\title{
Hard transition to chaotic dynamics in Alfvén wave fronts
}

\author{
J. R. Sanmartin, O. Lopez-Rebollal, and E. del Rio \\ Escuela Tecnica Superior de Ingenieros Aeronauticos, Universidad Politecnica de Madrid, Madrid, Spain \\ S. Elaskar \\ Departamento de Aeronautica, Universidad Nacional de Cordoba, Cordoba, Argentina
}

(Received 7 August 2003; accepted 4 February 2004; published online 15 April 2004)

\begin{abstract}
The derivative nonlinear Schrödinger (DNLS) equation, describing propagation of circularly polarized Alfvén waves of finite amplitude in a cold plasma, is truncated to explore the coherent, weakly nonlinear, cubic coupling of three waves near resonance, one wave being linearly unstable and the other waves damped. In a reduced three-wave model (equal dampings of daughter waves, three-dimensional flow for two wave amplitudes and one relative phase), no matter how small the growth rate of the unstable wave there exists a parametric domain with the flow exhibiting chaotic relaxation oscillations that are absent for zero growth rate. This hard transition in phase-space behavior occurs for left-hand (LH) polarized waves, paralleling the known fact that only LH time-harmonic solutions of the DNLS equation are modulationally unstable, with damping less than about (unstable wave frequency) $)^{2} / 4 \times$ ion cyclotron frequency. The structural stability of the transition was explored by going into a fully 3 -wave model (different dampings of daughter waves, four-dimensional flow); both models differ in significant phase-space features but keep common features essential for the transition. (C) 2004 American Institute of Physics.
\end{abstract}

[DOI: $10.1063 / 1.1691453]$

\section{INTRODUCTION}

Nonlinear Alfvén-wave interactions may be present in astrophysical, space and laboratory plasmas, with effects that range from heating to driving of current. A recent space example involves orbiting conductive tethers, which, if in electrical contact with the ionosphere, radiate Alfvén waves. ${ }^{1} \mathrm{~A}$ steady current flowing along a tether results in continual charge exchange with the ambient plasma, circuit closure being accomplished by charge-carrying Alfvén waves excited in the ionosphere by the passage of the system. Wave structures (called Alfvén wings) attached at or near both tether ends present an Airy-functions behavior if linearly described. $^{2}$ Nonlinear effects, which should appear at the near wave front, might be affected by the magnetic self-field generated by the very current of the tether. ${ }^{3}$

An Airy-like linear structure is a feature common in fronts of dispersive waves radiated by a moving source, as with ion-acoustic waves excited by a charged body moving mesothermally in a low ion-temperature plasma; the nonlinear wave front is then described by the Korteweg-de Vries equation. ${ }^{4}$ In the case of Alfvén waves, some strong nonlinear effects are known to be described by the derivative nonlinear Schrödinger (DNLS) equation, ${ }^{5}$ which admits soliton solutions ${ }^{6}$ and has proved amenable to the inverse scattering method for obtaining general solutions. ${ }^{7}$ A variety of behaviors allowed by the DNLS equation and its modifications have been analyzed. ${ }^{8}$

Here we show how the DNLS equation may also serve to describe weak nonlinear effects represented by the coherent coupling of a few waves, and we explore its complex dynamics. The local, coherent interaction of three waves at or near resonance (3WRI) is an ubiquitous feature of nonlin- ear mediums. The $3 \mathrm{WRI}$ is especially important in both unmagnetized and magnetized plasmas, where dispersive effects can keep nonlinearities weak and electromagnetic waves make coupling to external energy sources easy. The 3 WRI has been extensively studied and remains a basic nonlinear paradigm. ${ }^{9}$

The 3WRI evolution for lossless quadratic coupling, with a mode linearly unstable and the two other modes equally damped, is described by a three-dimensional (3D) flow of two wave amplitudes and one relative phase (a reduced 3-wave interaction). If damping rates exceed the growth rate $\Gamma$ of the unstable mode the system is attracted to point sets of vanishing 3D volume, and its long-time behavior may be chaotic. ${ }^{10-12}$ For small $\Gamma$, a consistent analysis of the flow using a multiple time-scales method, led to an 1D chaotic map. ${ }^{13}$ Actually, the system exhibits a hard transition to complex phase-space dynamics: no matter how small $\Gamma>0$ there exists a fully developed attractor that is absent at $\Gamma \leqslant 0$ and is chaotic for some parametric domain; this is an example of a broad scenario for chaos also present in the resonant coupling of two oscillators at frequency ratio 2:q, $q$ integer, with the first oscillator unstable. ${ }^{14}$ For quadratic coupling (corresponding to $q=1$ in the two-oscillator case), the hard transition and the effects of noise have been experimentally verified using electronic oscillators; ${ }^{15}$ also, the hard transition was found to persist when the daughter waves had unequal dampings, the flow then being $4 \mathrm{D}$ rather than $3 \mathrm{D} .^{16,17}$

Cubic interaction, corresponding to $q=2$ (or $1: 1$ frequency ratio) in the two-oscillator case, allows a variety of coupling structures. A reduced 3-wave truncation of the nonlinear Schrödinger equation showed chaotic behavior at finite 
$\Gamma ;{ }^{18}$ a hard transition was encountered in a two-oscillator model of a spherical swing. ${ }^{14,19}$ In the present paper we explore weakly nonlinear dynamics in a truncation of the DNLS equation that shows more complex cubic coupling, in both reduced and full 3-wave models. We want, in particular, to ascertain whether gross features in dynamical behavior, like fully developed phase-space attractors at $\Gamma=0^{+}$, are structurally stable, as suggested by their appearance for quadratic coupling in both 3D and 4D flows, and for the particular cubic coupling of Refs. 14 and 19.

Structural stability would be important because a 3WRI model may fail on a number of conditions it requires. Coherence is lost, leading to a random-phase approximation, when the interaction time exceeds the inverse frequency widths of the modes. ${ }^{20,21}$ The 3WRI model will also, strictly, fail for wave amplitudes so large that the interaction time drops to values comparable with wave periods. ${ }^{22}$ Dynamics just temporal, rather than spatiotemporal, may require long uniform wave trains or standing waves $;{ }^{23}$ multiple waves may be involved. $^{24,25}$

In Sec. II we present the reduced 3-wave model of the DNLS equation. In Sec. III we analytically determine the $\Gamma=0$ attractor of the system. In Sec. IV we derive analytical and numerical results on the small, positive $\Gamma$ attractor(s). The full 3-wave model (daughter waves with different dampings) is considered in Sec. V. A discussion is given in Sec. VI.

\section{REDUCED 3-WAVE MODEL OF THE DNLS EQUATION}

The derivative nonlinear Schrödinger equation describes the evolution of circularly polarized Alfvén waves of finite amplitude propagating along an unperturbed uniform magnetic field in a cold, homogeneous and lossless plasma. The description uses a two-fluid, quasineutral approximation (with electron inertia and current displacement neglected). Taking the unperturbed magnetic field $B_{0}$ in the $z$ direction, the DNLS equation reads ${ }^{5-8}$

$$
\frac{\partial \phi}{\partial t}+\frac{\partial}{\partial z}\left[\phi\left(1+\frac{|\phi|^{2}}{4}\right)\right] \pm \frac{i}{2} \frac{\partial^{2} \phi}{\partial z^{2}}+\hat{\gamma} \phi=0,
$$

where $\phi, t$, and $z$ are dimensionless perturbed field and variables,

$$
\phi \equiv \frac{B_{x} \pm i B_{y}}{B_{0}}, \quad \omega_{c i} t \rightarrow t, \quad \frac{\omega_{c i}}{V_{A}} z \rightarrow z,
$$

$\omega_{c i}$ is the ion cyclotron frequency and $V_{A}$ is the Alfvén velocity. The upper (lower) sign in Eqs. (1) and (2) corresponds to a left-hand (right-hand) circularly polarized wave propagating in the $z$ direction; $\hat{\gamma}$ would be some appropriate growth/damping linear operator. ${ }^{18}$ Equation (1) is derived under the following ordering scheme for perturbed quantities ( $n$ and $v_{z}$ are plasma density and velocity along the $z$ axis):

$$
\frac{B_{x}}{B_{0}} \approx \frac{B_{y}}{B_{0}} \approx \sqrt{\frac{n-n_{0}}{n_{0}}} \approx \sqrt{\frac{v_{z}}{V_{A}}} .
$$

To study weakly nonlinear interactions, we consider an approximate solution of Eq. (1) consisting of three traveling waves,

$$
\phi=2 \sum_{j=1}^{3} \phi_{j}(t) e^{i \lambda_{j}}, \quad \lambda_{j}=k_{j} z-\omega_{j} t,
$$

satisfying a resonance condition $2 k_{1}=k_{2}+k_{3}$. Wave number and frequency of modes are related by the linear (lossless) dispersion relation for circularly polarized Alfvén waves at low wave number, $\omega_{j}=k_{j} \mp k_{j}^{2} / 2$, or in dimensional form $\left(\omega \rightarrow \omega / \omega_{c i}, k \rightarrow k V_{A} / \omega_{c i}, k \equiv k_{z}>0\right)$,

$$
\omega_{j}=V_{A} k_{j}\left(1 \mp \frac{1}{2} \frac{V_{A} k_{j}}{\omega_{c i}}\right) .
$$

Both the growth/damping and the nonlinear term in (1) make the complex amplitudes $\phi_{j}$ vary slowly in time. Introducing Eq. (3) in (1) and considering only the $k_{1}, k_{2}$, and $k_{3}$ components one arrives at

$$
\begin{aligned}
\dot{\phi}_{1}+ & \gamma_{1} \phi_{1}+i k_{1}\left[\left(\left|\phi_{1}\right|^{2}+2\left|\phi_{2}\right|^{2}+2\left|\phi_{3}\right|^{2}\right) \phi_{1}\right. \\
& \left.+2 \phi_{1}^{*} \phi_{2} \phi_{3} e^{i \nu t}\right]=0, \\
\dot{\phi}_{2}+ & \gamma_{2} \phi_{2}+i k_{2}\left[\left(2\left|\phi_{1}\right|^{2}+\left|\phi_{2}\right|^{2}+2\left|\phi_{3}\right|^{2}\right) \phi_{2}\right. \\
& \left.+\phi_{1}^{2} \phi_{3}^{*} e^{-i \nu t}\right]=0, \\
\dot{\phi}_{3}+ & \gamma_{3} \phi_{3}+i k_{3}\left[\left(2\left|\phi_{1}\right|^{2}+2\left|\phi_{2}\right|^{2}+\left|\phi_{3}\right|^{2}\right) \phi_{3}\right. \\
& \left.+\phi_{1}^{2} \phi_{2}^{*} e^{-i \nu t}\right]=0,
\end{aligned}
$$

where $\dot{\phi}_{j}$ is $d \phi_{j} / d t$ and $\nu \equiv 2 \omega_{1}-\omega_{2}-\omega_{3}$ is a frequency mismatch. We assume that all other components, in particular those involving wave numbers $2 k_{2}-k_{1}, 2 k_{3}-k_{1}, 2 k_{2}$ $-k_{3}$, and $2 k_{3}-k_{2}$, arising from using (3) in the nonlinear term of Eq. (1), are strongly damped. ${ }^{18}$

Setting $\phi_{j}(t)=a_{j}(t) \exp \left[i \psi_{j}(t)\right]$ in Eqs. (5a) $-(5 \mathrm{c})$ with $a_{j}, \psi_{j}$ real, and using the resonance condition, the above three complex equations are reduced to four real equations,

$$
\begin{aligned}
\dot{a}_{1}= & -\gamma_{1} a_{1}-\left(k_{2}+k_{3}\right) a_{1} a_{2} a_{3} \sin \beta, \\
\dot{a}_{2}= & -\gamma_{2} a_{2}+k_{2} a_{1}^{2} a_{3} \sin \beta, \\
\dot{a}_{3}= & -\gamma_{3} a_{3}+k_{3} a_{1}^{2} a_{2} \sin \beta, \\
\dot{\beta}= & \nu+\left[a_{1}^{2}\left(k_{2} \frac{a_{3}}{a_{2}}+k_{3} \frac{a_{2}}{a_{3}}\right)-2\left(k_{2}+k_{3}\right) a_{2} a_{3}\right] \\
& \times \cos \beta-k_{2}\left[a_{1}^{2}-a_{2}^{2}\right]-k_{3}\left[a_{1}^{2}-a_{3}^{2}\right],
\end{aligned}
$$

where $\beta \equiv \pi+\nu t+\psi_{2}+\psi_{3}-2 \psi_{1}$.

At this point and throughout Secs. II-IV we restrict the analysis, for simplicity, to the case $\gamma_{2}=\gamma_{3} \equiv \gamma$ and return to the full 3-wave system in Sec. V. Multiplying Eq. (6b) by $2 k_{3} a_{2}$, Eq. (6c) by $2 k_{2} a_{3}$, and subtracting from each other there results

$$
\frac{d}{d t}\left(k_{3} a_{2}^{2}-k_{2} a_{3}^{2}\right)=-2 \gamma\left(k_{3} a_{2}^{2}-k_{2} a_{3}^{2}\right) ;
$$

Eq. (7) shows $k_{3} a_{2}^{2}-k_{2} a_{3}^{2}$ (but not $a_{2}^{2}$ or $a_{3}^{2}$ ) to decay exponentially with time. For a study of the long-time behavior of the system, we may then take $k_{3} a_{2}^{2}=k_{2} a_{3}^{2}$ from the outset. 
Note that the frequency mismatch is positive and negative for left-hand ( $\mathrm{LH})$ and right-hand $(\mathrm{RH})$ polarization, respectively, using Eq. (4) one finds in dimensional form

$$
\frac{\nu}{\omega_{c i}} \approx \pm\left(\frac{\omega_{1}}{\omega_{c i}}\right)^{2}\left(\frac{k_{2}-k_{3}}{k_{2}+k_{3}}\right)^{2} .
$$

This sign difference will later be shown to lead to fundamentally different dynamics for the two polarizations. Finally we may both take $k_{3}<k_{2}$ and equal signs for $a_{2}$ and $a_{3}$ with no loss of generality (for opposite signs, setting $\beta \rightarrow \pi+\beta$ would again leave the system invariant); also, we may take all three $a_{1}, a_{2}, a_{3}$ positive.

Writing $\gamma_{1} \equiv-\Gamma<0$ and introducing new variables

$$
\sqrt{k_{2} k_{3}} a_{1}^{2} \rightarrow a_{1}^{2}, \quad\left(k_{2}+k_{3}\right) \sqrt{k_{3} / k_{2}} a_{2}^{2} \rightarrow a_{2}^{2},
$$

system $(6 a)-(6 d)$ is reduced to three real nonlinear equations,

$$
\begin{aligned}
& \dot{a}_{1}=\Gamma a_{1}-a_{1} a_{2}^{2} \sin \beta, \\
& \dot{a}_{2}=-\gamma a_{2}+a_{1}^{2} a_{2} \sin \beta, \\
& \dot{\beta}=\nu-2\left(a_{1}^{2}-a_{2}^{2}\right)(\bar{V}-\cos \beta)-a_{2}^{2} / \bar{V},
\end{aligned}
$$

where

$$
\bar{V} \equiv \frac{1+k_{3} / k_{2}}{2 \sqrt{k_{3} / k_{2}}}>1, \quad\left(\frac{k_{3}}{k_{2}}<1\right) .
$$

The limit case $\bar{V}=1$ would exactly recover a truncation of the 1D nonlinear Schrödinger equation describing the parametric excitation of linearly damped waves by the oscillating two-stream instability in plasmas. ${ }^{18} \mathrm{We}$ also note that some resonant interactions of two oscillators with frequency ratio 1:1, which have been analyzed by López-Rebollal and Sanmartin, are described by system (9a)-(9c) with the last term in $(9 \mathrm{c})$ missing. ${ }^{14,19}$

\section{III. $\Gamma=0$ ATTRACTOR FOR THE REDUCED 3-WAVE MODEL}

In this section we discuss analytical results that can be readily obtained from system (9a)-(9c) and we determine its $\Gamma=0$ attractor. A trivial result concerns the flow divergence in (3D) phase-space $a_{1}^{2}, a_{2}^{2}$, and $\beta$, reading

$$
\frac{\partial}{\partial a_{1}^{2}} \frac{d a_{1}^{2}}{d t}+\frac{\partial}{\partial a_{2}^{2}} \frac{d a_{2}^{2}}{d t}+\frac{\partial}{\partial \beta} \frac{d \beta}{d t}=2(\Gamma-\gamma) .
$$

Nonlinear conservative coupling naturally preserves volume. For $\Gamma<\gamma$, as assumed here, the long-time attractor of the system will be a point-set of vanishing $3 \mathrm{D}$ volume.

Again from system (9a)-(9c) one obtains equations that would represent conservation laws in the no-dissipation limit,

$$
\begin{aligned}
& \frac{d}{d t}\left(a_{1}^{2}+a_{2}^{2}\right)=2 \Gamma a_{1}^{2}-2 \gamma a_{2}^{2}, \\
& \frac{d}{d t}\left[a_{2}^{2}\left(h_{0}-\frac{a_{2}^{2}}{2 \bar{V}}\right)\right]=2 \Gamma a_{2}^{2}\left(h_{0}-\nu\right)-2 \gamma a_{2}^{2}\left(h_{0}-\frac{a_{2}^{2}}{\bar{V}}\right),
\end{aligned}
$$

where

$$
h_{0}\left(a_{1}, \beta\right) \equiv \dot{\beta}\left(a_{2}=0\right)=\nu-2 a_{1}^{2}(\bar{V}-\cos \beta) .
$$

For $\Gamma=\gamma=0$, Eq. (11) rewritten in the original variables would be a Manley-Rowe relation for conservation of action density in wave packets that follow Hamiltonian dynamics. Equation (7) would be a second Manley-Rowe relation: since phases $\psi_{j}$ only enter Eqs. (6a)-(6d) through the combination $2 \psi_{1}-\psi_{2}-\psi_{3}$, there would be two cyclic angular coordinates in the full 3-wave case. Equation (12) with $\Gamma=\gamma=0$, in turn, can be shown to express energy conservation when the constant-action laws are taken into account.

We note next that Eq. (9b) shows the plane $a_{2}=0$ to be invariant. When combined with Eq. (12) it yields

$$
\begin{aligned}
\frac{d}{d t}\left[h_{0}-\frac{a_{2}^{2}}{2 \bar{V}}\right]= & -2 a_{1}^{2} \sin \beta \times\left[h_{0}-\frac{a_{2}^{2}}{2 \bar{V}}\right] \\
& +\gamma \frac{a_{2}^{2}}{\bar{V}}+2 \Gamma\left(h_{0}-\nu\right) .
\end{aligned}
$$

In the conservative case, the surface $h_{0}\left(a_{1}, \beta\right)-a_{2}^{2} / 2 \bar{V}=0$, which only exists for frequency mismatch $\nu>0$, i.e., for $\mathrm{LH}$ polarization, would be invariant. In what follows we will only consider LH polarization.

For $\Gamma<0$, Eq. (11) proves the equilibrium state $a_{1}=a_{2}$ $=0$ to be a global attractor. This equilibrium is unstable for $\Gamma>0$. In this section we consider the long-time attractor of system $(9 a)-(9 c)$ at $\Gamma=0$. Note that the entire flow is now asymptotic to the surface $a_{2}=0$, because $a_{1}^{2}+a_{2}^{2}$ will keep diminishing in Eq. (11) unless $a_{2}$ vanishes. Since that surface is invariant, trajectories should be asymptotic to its critical elements with transverse stable manifolds.

Consider then the flow on $a_{2}=0$ at $\Gamma=0$, Eq. (9a) then yielding $a_{1}=$ constant. The intersection of the plane $a_{2}=0$ and the cylindrical surface $h_{0}\left(a_{1}, \beta\right)=0$ is a line $\Lambda$ of fixed points,

$$
\begin{aligned}
& a_{2}=0, \\
& h_{0} \equiv \nu-2 a_{1}^{2}(\bar{V}-\cos \beta)=0 .
\end{aligned}
$$

Figure 1 shows both $\Lambda$ and the surface $h_{0}=0$ for $\bar{V}>1 ; \Lambda$ would reach up to infinity for $\bar{V}=1$. Linearizing the vector field at the fixed points we find eigenvalues $\lambda_{1}=-2 a_{1}^{2}$ $\times \sin \beta$, and $\lambda_{2}=0$, for eigenvectors tangent to the line $a_{1}$ $=$ constant through the corresponding fixed point, and tangent to $\Lambda$, respectively. From the sign of $\lambda_{1}$ it follows that, for flow on $a_{2}=0, \Lambda$ points with $\beta<\pi$ are stable and $\beta>\pi$ points are unstable; two $a_{1}=$ constant heteroclinic orbits join each symmetric pair of $\Lambda$ points.

The third eigenvalue is clearly the factor multiplying $a_{2}$ in Eq. (9b), $\lambda_{3}=-\gamma+a_{1}^{2} \sin \beta$, with the associated eigenvector parallel to the $a_{2}$ axis. It follows that for motion off $a_{2}$ $=0$ the $\beta>\pi$ branch is stable, whereas in the branch $\beta<\pi$, under condition

$$
\bar{V}^{2}<1+(\nu / 2 \gamma)^{2}
$$

there are points $P_{0}$ and $P_{0}^{*}$ that have $\lambda_{3}=0$ and are given by

$$
a_{1}^{2}=\frac{\gamma}{\sin \beta}=\frac{\bar{V} \nu \mp \sqrt{\nu^{2}-4 \gamma^{2}\left(\bar{V}^{2}-1\right)}}{2\left(\bar{V}^{2}-1\right)}
$$




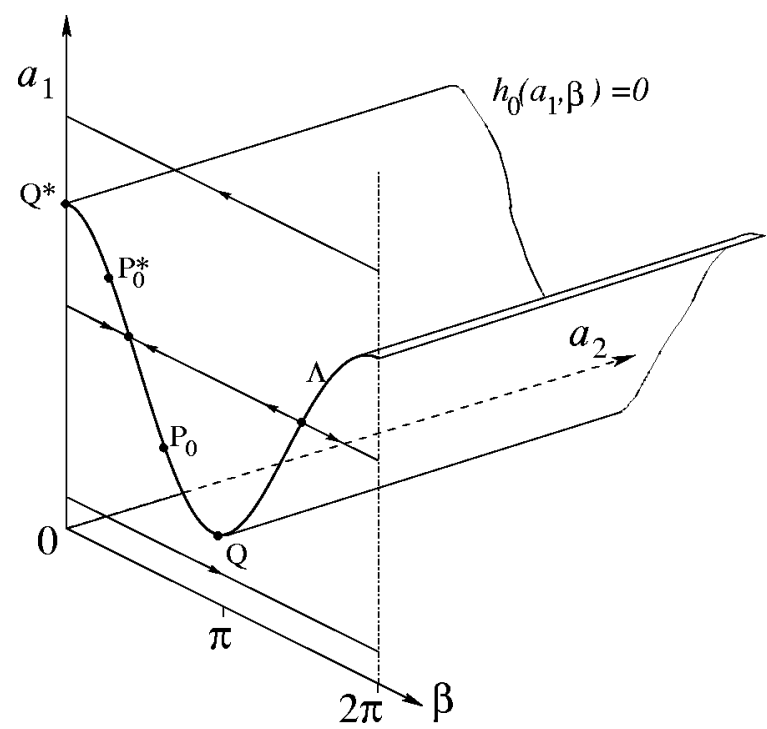

FIG. 1. Line $\Lambda$ of fixed points on invariant plane $a_{2}=0$ at $\Gamma=0$, and periodic orbits above and below; for $\beta<\pi$ only the $\operatorname{arcs} Q P_{0}$ and $P_{0}^{*} Q^{*}$ are stable off $a_{2}=0$. Also shown is the cylindrical surface $h_{0}\left(a_{1}, \beta\right)=0$.

for - and + signs, respectively, with $\beta\left(P_{0}^{*}\right)<\pi / 2$ always, but $\beta\left(P_{0}\right)>\pi / 2$ for $\nu / 2 \gamma>\bar{V}$. Only $\Lambda$ points in the arc $P_{0} P_{0}^{*}$ are unstable off $a_{2}=0$. For the flow in the entire 3D space the stable fixed points are those in the $\beta<\pi$ branch of $\Lambda$ below $P_{0}$ and above $P_{0}^{*}$. Note that $a_{1}\left(P_{0}^{*}\right) \rightarrow \infty$ as $\bar{V}$ $\rightarrow 1$.

In the plane $a_{2}=0$ there is another type of critical elements. There are periodic orbits that move below the bottom $Q$ of $\Lambda$ at constant $a_{1}<a_{1 Q}$, from $\beta=0$ to $\beta=2 \pi$, and that are described by Eq. (9c) now reading $\dot{\beta}=h_{0}\left(a_{1}, \beta\right)$; again, there are periodic orbits above $Q^{*}$ in Fig. 1. [Their period is $\pi /\left[\left(\bar{V} a_{10}^{2}-\nu / 2\right)^{2}-a_{10}^{4}\right]^{1 / 2}$, which diverges for $a_{10}$ $=a_{1 Q}\left(a_{10}=a_{1 Q^{*}}\right)$, when the periodic orbit becomes an homoclinic trajectory at $Q\left(Q^{*}\right)$, as seen in the figure.] Clearly, $a_{2}=0$ perturbations of any such orbits leave the system moving in another nearby orbit. Also, all these periodic orbits are stable off $a_{2}=0$ : At vanishing $a_{2}$ we have $\dot{\beta}$ $=\mathrm{O}(1)$ whereas $a_{1}$ changes at a rate of order $a_{2}^{2}$; taking $\mathrm{d}\left(\ln a_{2}\right) / \mathrm{d} t$ from $(9 \mathrm{~b})$, its average over a period is $-\gamma<0$, the contribution of the $\sin \beta$ term vanishing.

Under condition (16) one may say that the stable arc $Q P_{0}$ and the periodic orbits below $Q$ make up one attractor of the flow and the stable arc $P_{0}^{*} Q^{*}$ and the periodic orbits above $Q^{*}$ make up a second attractor. There is a fundamental difference between these two attractors however. Since $\Lambda$ points in the $\operatorname{arc} P_{0} P_{0}^{*}$ have an 1D unstable manifold transverse to $a_{2}=0$ there exist singular, heteroclinic orbits that leave this plane at those points, and return to it at a lower $a_{1}$, as seen from Eq. (11) with $\Gamma=0$. Equation (14) with $\Gamma=0$ proves that an orbit leaving $\Lambda$ between $P_{0}$ and $P_{0}^{*}$ has the quantity $h_{0}-a_{2}^{2} / 2 \bar{V}$, and therefore $h_{0}$ itself, non-negative thereafter, corresponding to it moving below the cylindrical surface $h_{0}\left(a_{1}, \beta\right)=0$ (Fig. 1). The singular orbit may reach a point in the arc $Q P_{0}$ from the left, keeping $\beta<\pi$ throughout, or may approach the set of periodic orbits. It may also pass just below the surface $h_{0}\left(a_{1}, \beta\right)=0$ to reach the range $\beta>\pi$ still off the plane $a_{2}=0$, making $\dot{a}_{1}$ positive in Eq. (9a); the orbit will then emerge at $\beta=0$ with $a_{1}>a_{1 Q}$ and again reach a point in the arc $Q P_{0}$ from the left.

\section{IV. $\Gamma \rightarrow 0^{+}$ATTRACTOR}

When $\Gamma$ is made positive under condition (16), assumed here, there are just two fixed points, $P$ and $P^{*}$, given by equations

$$
\begin{aligned}
& \nu \sin \beta=2(\gamma-\Gamma)(\bar{V}-\cos \beta)+\Gamma / \bar{V}, \\
& \gamma / a_{1}^{2}=\sin \beta, \\
& \Gamma / a_{2}^{2}=\sin \beta,
\end{aligned}
$$

which recover (17) for $P_{0}$ and $P_{0}^{*}$ as $\Gamma \rightarrow 0$. The characteristic equation for the stability of those two points is

$$
\begin{aligned}
& (\lambda+2 \gamma-2 \Gamma)\left(\lambda^{2}+4 \gamma \Gamma\right) \\
& \quad+\frac{2 \gamma \Gamma}{\tan \beta}\left[\frac{\lambda}{\sin \beta}\left\{\frac{1}{\bar{V}}-4(\bar{V}-\cos \beta)\right\}-2 \nu\right]=0 .
\end{aligned}
$$

For $\Gamma=0$, Eq. (19) again recovers the values $\lambda_{1}=-2 \gamma, \lambda_{2}$ $=\lambda_{3}=0$ of Sec. III. For $\Gamma$ positive and small, one finds to order $\sqrt{ } \Gamma$,

$$
\lambda_{2,3}\left(P^{*}\right) \approx \pm \sqrt[4]{\nu^{2}-4 \gamma^{2}\left(\bar{V}^{2}-1\right)} \times \sqrt{2 \Gamma} \times a_{1}\left(P_{0}^{*}\right) ;
$$

$P^{*}$ at small $\Gamma$ is thus a saddle node with a $1 \mathrm{D}$ unstable manifold.

For the stability of $P$ we must go to order $\Gamma$,

$$
\begin{aligned}
& \lambda_{2,3}(P) \approx \pm i \sqrt[4]{\nu^{2}-4 \gamma^{2}\left(\bar{V}^{2}-1\right)} \times \sqrt{2 \Gamma} \times a_{1}\left(P_{0}\right)+\bar{\lambda} \Gamma, \\
& \bar{\lambda} \equiv \frac{1}{2 \tan \beta}\left(\frac{\nu}{\gamma}-\frac{1}{\bar{V} \sin \beta}\right) .
\end{aligned}
$$

The sign of $\bar{\lambda}$ is obtained by taking $\beta(\nu / \gamma, \bar{V})$ from Eq. (17) with the upper sign. We find that $P$, which only exists under condition (16) and is defined for $\bar{V}>1$, is stable above the line $(\nu / 2 \gamma)^{2}=\bar{V}^{2}$ [with $\beta\left(P_{0}\right)>\pi / 2$ as noticed in Sec. III] and below the line

$$
\left(\frac{\nu}{2 \gamma}\right)^{2}=\frac{1}{8 \bar{V}^{2}-4 \bar{V}^{4}-1} \quad\left(\text { for } \bar{V}^{2}<\frac{3}{2}\right)
$$

in the parametric plane $(\nu / 2 \gamma)^{2}, \bar{V}^{2}$. Point $P$ goes through a Hopf bifurcation, $\bar{\lambda}$ becoming positive, when crossing either line. Figure 2 summarizes the stability of $P$ for $\Gamma \rightarrow 0^{+}$. Figure 3 uses Eqs. (8) and (10) to represent stability domains in terms of parameters $k_{3} / k_{2}$ and $\omega_{c i} \gamma / V_{A}^{2} k_{1}^{2}$, where $V_{A} k_{1}$ $\approx \omega_{1}$.

Now consider the long-time behavior of the system for $\Gamma$ very small. Away from the surface $a_{2}=0$ the flow will closely follow $\Gamma=0$ trajectories. If a trajectory approaches a periodic orbit above $Q^{*}$, the term $\Gamma a_{1}$ in Eq. (9a) will make $a_{1}$ ultimately diverge, as the system slowly drifts through the set of periodic orbits; if the $\Gamma=0$ trajectory approaches the $\operatorname{arc} P_{0}^{*} Q^{*}$, the system will first have $a_{1}$ slowly rising along and very close to $\Lambda$, until reaching the set of periodic orbits 


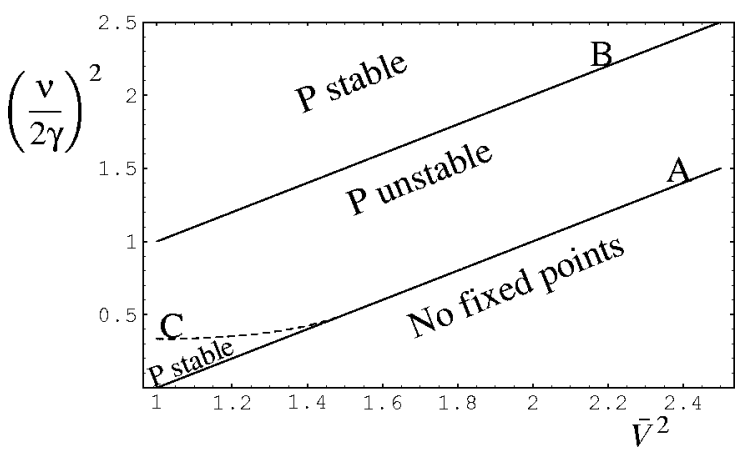

FIG. 2. Stability of fixed point $P$ at $\Gamma \rightarrow 0^{+}$, in parametric plane $(\nu / 2 \gamma)^{2}$, $\bar{V}^{2}$. Lines $A,(\nu / 2 \gamma)^{2}=\bar{V}^{2}-1 ; B,(\nu / 2 \gamma)^{2}=\bar{V}^{2} ; C$, given by Eq. (22).

at point $Q^{*}$. The case for $\Gamma=0$ trajectories approaching either the arc $Q P_{0}$ or the periodic orbits below is dramatically different.

Consider flow in the vicinity of the $\Gamma=0$ heteroclinic orbit corresponding to a $\Lambda$-point $M$ on the $P_{0} P_{0}^{*}$ arc, in the approach back to the surface $a_{2}=0$, below $P_{0}$. If the orbit approaches some point $m$ between $Q$ and $P_{0}$ and because of the term $\Gamma a_{1}, a_{1}$ should eventually start growing at rate $\Gamma$, keeping close to $\Lambda$. In terms of the eigenvalue $\lambda_{3}$ of Sec. III, Eq. (9b) can be written as $\mathrm{d} a_{2} / \mathrm{d} t=\lambda_{3} a_{2}$; since $\lambda_{3}$ is negative for $\Lambda$ points below $P_{0}$ and positive from $P_{0}$ to $P_{0}^{*}$, and the $a_{1}$ rise takes times of order $1 / \Gamma, a_{2}$ will become exponentially small $\left(-\ln a_{2} \sim 1 / \Gamma\right)$. Once $P_{0}$ is reached, however, $a_{2}$ will start growing; when values $a_{2} \sim \sqrt{ } \Gamma$ are attained, $a_{1}$ can finally reach a maximum $M^{\prime}$ below $P_{0}^{*}$, and the trajectory again start separating from $\Lambda$. If the heteroclinic orbit approaches some periodic orbit below $Q, a_{1}$ will first slowly increase while the system drifts among the lower set of periodic orbits to reach $\Lambda$.

In the parametric domain of Fig. 2 where $P$ is stable, trajectories starting within some bassin of attraction in phase space have a sequence of points $M, m, M^{\prime}, \ldots$, converging to point $P$ as given, to lowest order in $\Gamma$, by Eqs. (17) and $a_{2}^{2}$ $=\Gamma \times a_{1}^{2} / \gamma$. The general attractor structure following the loss of stability of $P$ at crossing line $B$ (or $C$ ) at fixed $\bar{V}$, giving rise to a limit cycle, depends on the value of $\bar{V}$. At $\bar{V}$ very close to unity the set of periodic orbits is rarely involved in the attractor. Figure 4 shows a limit-cycle attractor for $\bar{V}$

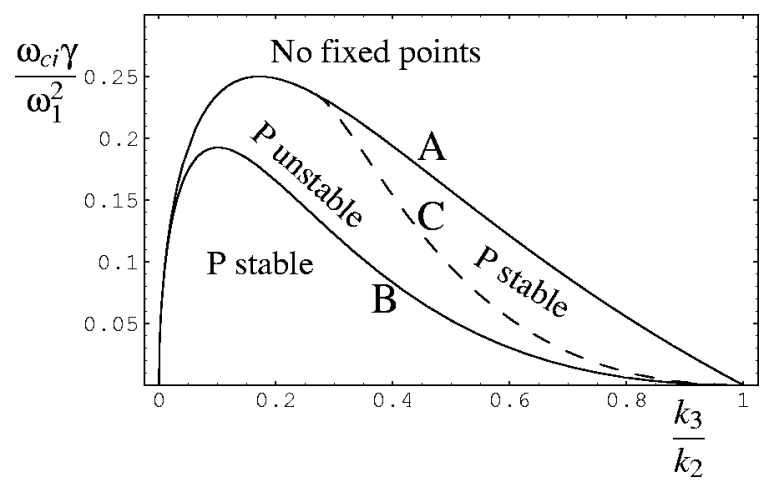

FIG. 3. Stability of fixed point $P$ at $\Gamma \rightarrow 0^{+}$, in parametric plane $\omega_{\text {ci }} \gamma / V_{A}^{2} k_{1}^{2}$, $k_{3} / k_{2}$.

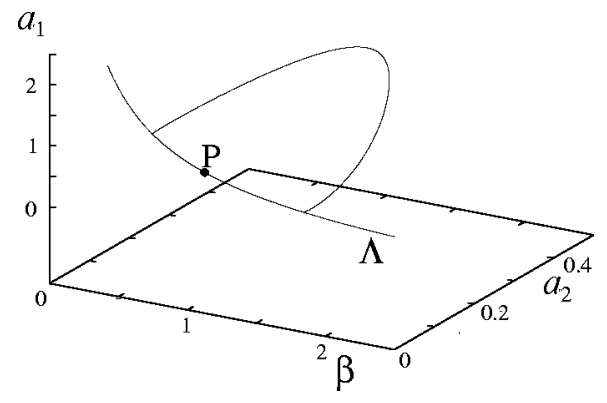

FIG. 4. Limit 1-cycle attractor for $\bar{V}=61 / 60\left(k_{3} / k_{2}=25 / 36\right), \Gamma / \gamma=0.001$, $\nu / \gamma=1.3$, setting $\gamma=1$.

$=61 / 60\left(k_{3} / k_{2}=25 / 36\right), \quad \Gamma / \gamma=0.001$ and $\nu / \gamma=1.3$ (with $\gamma=1$ ), which is determined by numerically following a single trajectory for long times; Fig. 5 shows a map for the attractor of Fig. 4. Figure 6 shows a chaotic attractor for the same values of $\bar{V}$ and $\Gamma / \gamma$, and $\nu / \gamma=2.0$. Periodic orbits are usually involved at greater $\bar{V}$. Figures 7 and 8 show the lower parts of a limit 2-cycle attractor and of a chaotic attractor projected in the $a_{1}, \beta$ plane, for $\bar{V}=13 / 12\left(k_{3} / k_{2}=4 / 9\right)$, $\Gamma / \gamma=0.001$ and two values of $\nu / \gamma$. For $\bar{V}^{2}>3 / 2\left(k_{3} / k_{2}<2\right.$ $-\sqrt{ } 3$ ), and above but close to line $A$ in Fig. 2 , the $\operatorname{arc} P P_{0}$ is short, leaving $a_{2}$ still exponentially small when the system reaches $P_{0}^{*}$; with $a_{2}$ decreasing and $\dot{a}_{1}$ remaining positive thereafter, $a_{1}$ will diverge, as in the case of trajectories approaching the $\operatorname{arc} P_{0}^{*} Q^{*}$.

Consider the limit cycle in Fig. 4. In general, a $\Gamma \rightarrow 0^{+}$ attractor nested somehow around point $P$ may be described by an exact 1D map representing every maximum of $a_{1}\left(a_{1 M^{\prime}}\right)$ in a trajectory within its bassin of attraction, versus the preceding maximum $\left(a_{1 M}\right)$. This map can be determined by a two-step algorithm. In the first step, one numerically follows the heteroclinic orbit from any point $M$ in the $P_{0} P_{0}^{*}$ arc of $\Lambda$ to the corresponding point $m$ below $P_{0}$. The second step is the rise on $\Lambda$ at vanishing rate $(\Gamma \rightarrow 0, t$ $\sim 1 / \Gamma$ ) up to the next maximum $M^{\prime}$, which can be analytically determined by noting that, no matter how close the solution to a heteroclinic $M \rightarrow m$ orbit, Eq. (9a) will ultimately read $d a_{1} / d t=\Gamma a_{1}$.

Using $\mathrm{d} a_{2} / \mathrm{d} t=\lambda_{3} a_{2}$, one obtains

$$
\frac{a_{1}^{2} \sin \beta-\gamma}{a_{1}} d a_{1}=\Gamma d \ln a_{2},
$$

with $a_{1}$ and $\beta$ related through the equation $h_{0}\left(a_{1}, \beta\right) \equiv 0$. The integral of the left-hand side above, for the entire rise from $m$ to $M^{\prime}$ [with $\ln \left(1 / a_{2}\right)$ small compared with $1 / \Gamma$ at either end], will vanish in the limit $\Gamma \rightarrow 0^{+}$. We thus find an equation relating points $m$ and $M^{\prime}$ for the slow rise on $\Lambda$,

$$
\begin{aligned}
& G\left(\zeta_{M^{\prime}}, \bar{V}, \frac{\gamma}{\nu} \sqrt{\bar{V}^{2}-1}\right)=G\left(\zeta_{m}, \bar{V}, \frac{\gamma}{\nu} \sqrt{\bar{V}^{2}-1}\right), \\
& \zeta \equiv\left(\bar{V}^{2}-1\right) \frac{2}{\nu} a_{1}^{2}-\bar{V}
\end{aligned}
$$




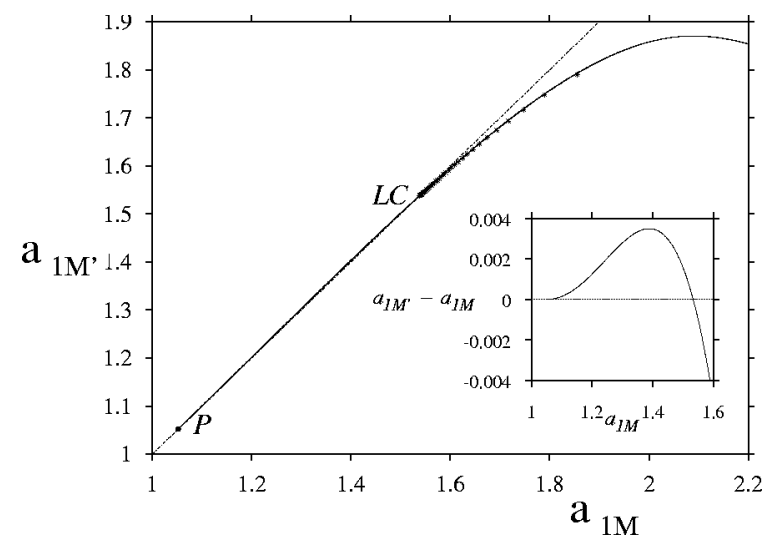

FIG. 5. 1D map of $a_{1 M}$ maxima for the attractor of Fig. 4, with fixed points at the fixed point $P$ of the flow, unstable, and at the $a_{1 M}$ maximum of the limit cycle (LC), stable. The crosses correspond to the sequence of maxima determined numerically on a single trajectory; the continuous line is the exact $\Gamma \rightarrow 0^{+}$map. The inset shows another view of the limit map.

$$
\begin{aligned}
G \equiv & \sqrt{1-\zeta^{2}}+\bar{V} \sin ^{-1} \zeta-\sqrt{\bar{V}^{2}-1} \sin ^{-1}\left(\frac{1+\bar{V} \zeta}{\bar{V}+\zeta}\right) \\
& -\frac{2 \gamma \sqrt{\bar{V}^{2}-1}}{\nu} \ln (\bar{V}+\zeta) .
\end{aligned}
$$

Figure 5 shows this $\Gamma \rightarrow 0^{+}$limit map of the sequence of maxima $a_{1 M}$ for the values of $\bar{V}, \nu$, and $\gamma$ of Fig. 4. The map has two fixed points, where it cuts the diagonal. One (unstable) fixed point of the map is the fixed point of the flow $P \approx P_{0}$, lying at $a_{1}$ just above unity. The second (stable) fixed point of the map corresponds to the maximum in the limit cycle, lying at $a_{1}$ just above 1.5 . Between both fixed points the map lies barely above the diagonal. Figure 5 also shows, to the right of the limit-cycle maximum, the approaching sequence of maxima for a single trajectory, at the values of $\bar{V}, \nu$, and $\gamma$ indicated, with $\Gamma / \gamma=0.001$, in good agreement with the limit map; this is more clearly seen in the zoom of the inset.

The inset of Fig. 6 shows the lower part of the chaotic attractor projected on the plane $a_{1}, \beta$. The inset shows clearly a fork in the attractor, with the trajectory sometimes approaching the $\beta<\pi$ branch of $\Lambda$ from the right. This is possible because the last term in Eq. (14) can make $h_{0}\left(a_{1}, \beta\right)$ negative when $a_{2}$ reaches down to values of order of $\Gamma$, the trajectory crossing above the cylindrical surface $h_{0}\left(a_{1}, \beta\right)=0$.

Consider now Figs. 7 and 8. If the $\Gamma=0$ heteroclinic orbit leaving some point $M$ in the $\operatorname{arc} P_{0} P_{0}^{*}$ approaches a periodic orbit below $Q$ with $a_{1}=a_{1 m}$, we will first have $a_{1}$ slowly increasing while the system drifts among the lower set of periodic orbits to reach $\Lambda$. During this rise Eq. (9c) will read $\dot{\beta}=h_{0}\left(a_{1}, \beta\right)=\mathrm{O}(1)$. With the contribution of the $\sin \beta$ term in (9b) averaging out during that stage, the overall relation between $m$ and $M^{\prime}$ would come out to be

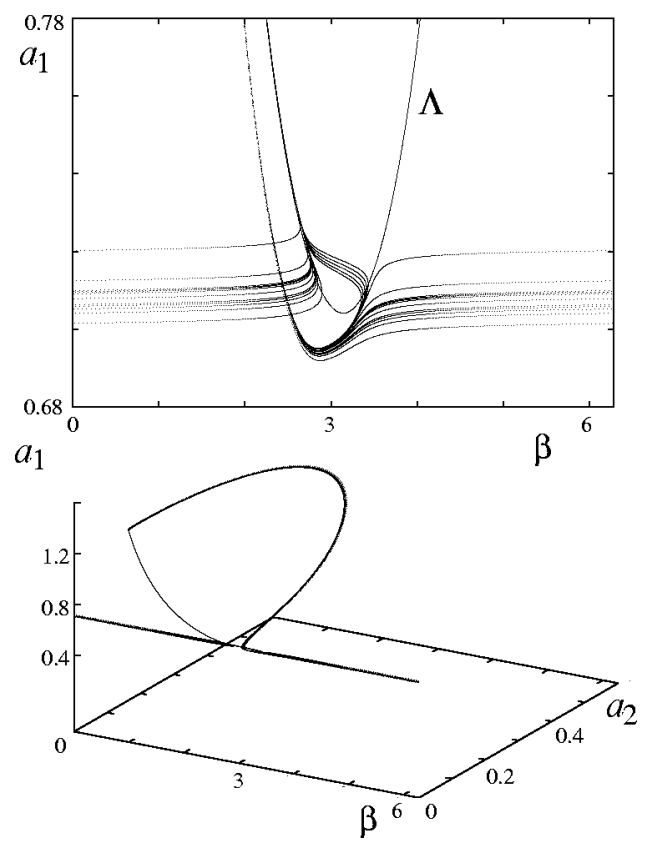

FIG. 6. Chaotic attractor for $\bar{V}=61 / 60\left(k_{3} / k_{2}=25 / 36\right), \Gamma / \gamma=0.001$, and $\nu / \gamma=2.0$ with $\gamma=1$. Also shown is the lower part of the attractor projected on the $a_{1}-\beta$ plane.

$$
\begin{aligned}
G\left(\zeta_{M^{\prime}}, \bar{V}, \frac{\gamma}{\nu} \sqrt{\bar{V}^{2}-1}\right)= & -\frac{\pi}{2}\left(\bar{V}-\sqrt{\bar{V}^{2}-1}\right) \\
& -\frac{2 \gamma \sqrt{\bar{V}^{2}-1}}{\nu} \ln \left(\bar{V}+\zeta_{m}\right) .
\end{aligned}
$$

\section{FULL 3-WAVE MODEL OF THE DNLS EQUATION}

We now briefly consider how changing to a full 3-wave model affects the dynamics of the system. In Eqs. (6a)-(6d) we set

$$
a_{1}^{2} \rightarrow \frac{a_{1}^{2}}{\sqrt{k_{2} k_{3}}}, \quad a_{2}^{2} \rightarrow \frac{a_{2}^{2}}{k_{2}+k_{3}} \sqrt{\frac{k_{2}}{k_{3}}},
$$

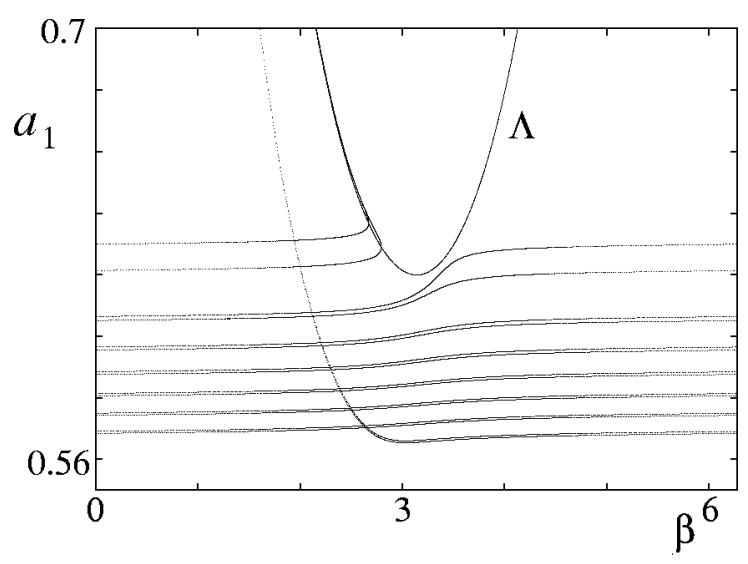

FIG. 7. Lower part of limit 2-cycle attractor projected on the $a_{1}-\beta$ plane, for $\bar{V}=13 / 12\left(k_{3} / k_{2}=4 / 9\right), \Gamma / \gamma=0.001$, and $\nu / \gamma=1.60122$ with $\gamma=1$. 


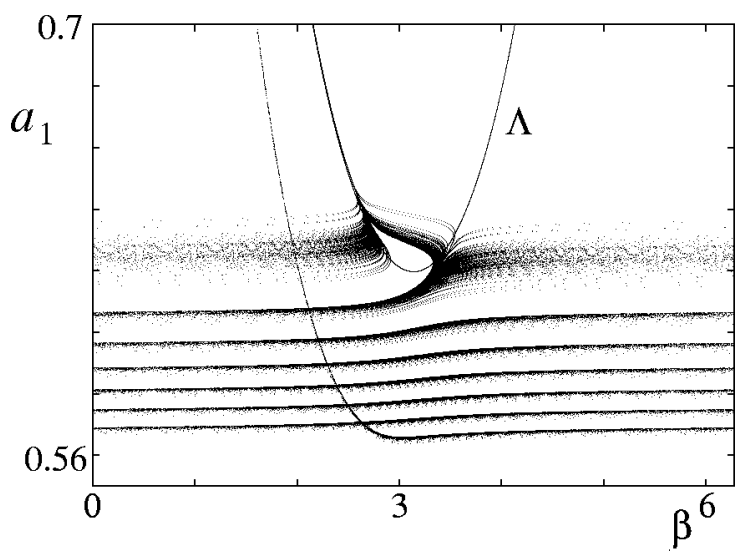

FIG. 8. Lower part of chaotic attractor projected on the $a_{1}-\beta$ plane, for $\bar{V} \approx 13 / 12\left(k_{3} / k_{2}=4 / 9\right), \Gamma / \gamma=0.001, \nu / \gamma=1.6$, and $\gamma=1$.

$$
a_{3}^{2} \rightarrow \frac{a_{3}^{2}}{k_{2}+k_{3}} \sqrt{\frac{k_{3}}{k_{2}}},
$$

and, assuming $\gamma_{2}<\gamma_{3}$, introduce a new variable,

$$
r \equiv a_{3} / a_{2},
$$

to replace $a_{3}$. We then find

$$
\begin{aligned}
\dot{a}_{1}= & \Gamma a_{1}-r a_{1} a_{2}^{2} \sin \beta, \\
\dot{a}_{2}= & -\gamma_{2} a_{2}+r a_{1}^{2} a_{2} \sin \beta, \\
\dot{r}= & -\left(\gamma_{3}-\gamma_{2}\right) r+\left(1-r^{2}\right) a_{1}^{2} \sin \beta, \\
\dot{\beta}= & \nu-2 a_{1}^{2}\left(\bar{V}-\frac{1+r^{2}}{2 r} \cos \beta\right)-2 r a_{2}^{2} \\
& \times \cos \beta+\frac{a_{2}^{2}}{2 \bar{V}}\left(\frac{k_{2}}{k_{3}}+r^{2} \frac{k_{3}}{k_{2}}\right),
\end{aligned}
$$

with $\bar{V}(<1)$ and $k_{3} / k_{2}$ still related by Eq. (10), where we do not need $k_{3} / k_{2}<1$. For $\gamma_{3}=\gamma_{2}$ the solution $r=1$ of Eq. (23c) would recover system (9a)-(9c) in Eqs. (23a), (23b), and $(23 \mathrm{~d})$. Note also that if a trajectory reaches the surface $r=1, r$ will remain less than unity thereafter in Eq. (23c); the $3 \mathrm{D}$ space $a_{2}=0$ will then be invariant.

If damping is resistive one has $\gamma=$ resistivity $\times \epsilon_{0} c^{2} k^{2}$ or, in dimensionless form (setting $\gamma \rightarrow \gamma \times \omega_{c i}, \quad k \rightarrow k$ $\left.\times \omega_{c i} / V_{A}\right), \quad \gamma \approx k^{2} / 2 \omega_{c e} \tau_{e}$, where $\omega_{c e}$ and $\tau_{e}$ are electron cyclotron frequency and Braginskii collision time, respectively. ${ }^{26}$ We then have $\gamma_{3} / \gamma_{2}=\left(k_{3} / k_{2}\right)^{2}$ with $k_{3}$ $>k_{2}$. For near-parallel propagation at angle $\theta \ll \sqrt{ } 2 \sqrt{ } \omega / \omega_{c i}$ and nonzero electron temperature $T_{e}$, we may have Landau damping, reading in dimensionless form, $\gamma=k \theta^{2} \times\left(m_{e} / m_{i}\right)$ $\times\left(\pi k T_{e} / 32 m_{e} V_{A}^{2}\right)^{1 / 2} \times \exp \left(-m_{e} V_{A}^{2} / 2 k T_{e}\right) .{ }^{27} \quad$ Now, $\gamma_{3} / \gamma_{2}$ $=k_{3} / k_{2}$, with $k_{3}>k_{2}$ again.

As in Sec. III, the long-time attractor of the system will be a point set of vanishing (now 4D) volume, which can be readily determined for $\Gamma=0$. First, Eq. (11) is recovered from (23a) and (23b), meaning that (for $\Gamma=0$ ) the entire flow is asymptotic to the space $a_{2}=0$, which is an invariant surface, trajectories being asymptotic to its critical elements with transverse stable manifolds.
Next, we consider the flow on $a_{2}=0$, where $a_{1}$ is now constant in (23a). There exists a line of fixed points $\Lambda$ given by the equations

$$
\begin{aligned}
& \left(\gamma_{3}-\gamma_{2}\right) r=a_{1}^{2}\left(1-r^{2}\right) \sin \beta, \\
& \nu r=a_{1}^{2}\left[2 \bar{V} r-\left(1+r^{2}\right) \cos \beta\right] .
\end{aligned}
$$

Eliminating $a_{1}$ in (24a) and (24b) yields a relation between $\beta$ and $r$ that can be written as

$$
\cos \left[\beta-\beta^{*}(r)\right]=\frac{2 \bar{V} r}{\Delta},
$$

where we defined

$$
\begin{aligned}
& \beta^{*} \equiv \cos ^{-1} \frac{1+r^{2}}{\Delta}, \quad \Delta \equiv \sqrt{\left(1+r^{2}\right)^{2}+\left(1-r^{2}\right)^{2} \bar{\nu}^{2}}, \\
& \bar{\nu} \equiv \frac{\nu}{\gamma_{3}-\gamma_{2}} .
\end{aligned}
$$

A solution to Eq. (25) for $\beta(r)$ only exists if the RHS does not exceed unity, requiring

$$
\begin{aligned}
& 0<r \leqslant r_{\max }<1, \quad \text { or } 1 / r_{\max } \leqslant r<\infty, \\
& r_{\max }^{2} \equiv \alpha-\sqrt{\alpha^{2}-1}, \quad \alpha \equiv \frac{\bar{\nu}^{2}+2 \bar{V}^{2}-1}{\bar{\nu}^{2}+1}>1 ;
\end{aligned}
$$

only for $\bar{V}=1$, (i.e., $k_{3}=k_{2}$ making $\alpha=1$ ), would $r$ reach unity. The line of fixed points has therefore two branches, $\Lambda_{l}$ for $r<1$ and $\Lambda_{h}$ for $r>1$.

For branch $\Lambda_{l}, \beta_{l}(r)$ is given by

$$
\beta_{l}=\cos ^{-1} \frac{1+r^{2}}{\Delta} \pm \cos ^{-1} \frac{2 \bar{V} r}{\Delta} \text {. }
$$

The plus sign in Eq. (27) applies as $a_{1}$ is increased from 0 to $a_{1}\left(r_{\max }\right)$, given as

$$
a_{1}\left(r_{\max }\right) / \sqrt{\nu}=\sqrt{\left(1+\bar{\nu}^{2}\right) \bar{V} / 2 \bar{\nu}^{2}\left(\bar{V}^{2}-1\right)} ;
$$

$\beta_{l} \quad$ decreases from $\cos ^{-1}\left(1 / \sqrt{1+\bar{\nu}^{2}}\right)+\pi / 2$ to $\cos ^{-1}\left(\sqrt{1+\bar{\nu}^{2} / \bar{V}^{2}} / \sqrt{1+\bar{\nu}^{2}}\right)$; and $r$ increases from 0 to $r_{\max }$. For greater $a_{1}$ the minus sign applies in (27), and $r$ decreases with increasing $a_{1}$; as $a_{1} \rightarrow \infty$ one finds $\beta_{l} \rightarrow 0$ and

$$
r \rightarrow r_{\infty} \equiv \bar{V}-\sqrt{\bar{V}^{2}-1} .
$$

Note that both $a_{1}$ and $\beta_{l}$ are double-valued functions of $r$ between $r_{\infty}$ and $r_{\max }$. For branch $\Lambda_{h}$ we have $\beta_{h}(r>1)$ $=2 \pi-\beta_{l}(1 / r)$ and $a_{1 h}(r>1)=a_{1 l}(1 / r)$. Figure 9 shows the projection of the line of fixed points on the $a_{1}-r$ and $\beta-r$ planes. It may be shown that throughout the branch $\Lambda_{l}$ the right-hand side of Eq. (24b) is positive; $\Lambda_{l}$ thus only exists for LH polarization.

Three eigenvalues of the linearized vector field at the fixed points have eigenvectors tangent to the invariant space $a_{2}=0$, determining the stability of flow on it,

$$
\lambda_{1,2}=\left(\gamma_{3}-\gamma_{2}\right) \frac{r^{2}+1}{r^{2}-1} \pm i\left(\nu-2 a_{1}^{2} \bar{V}\right) \frac{r^{2}-1}{r^{2}+1}, \quad \lambda_{3}=0,
$$




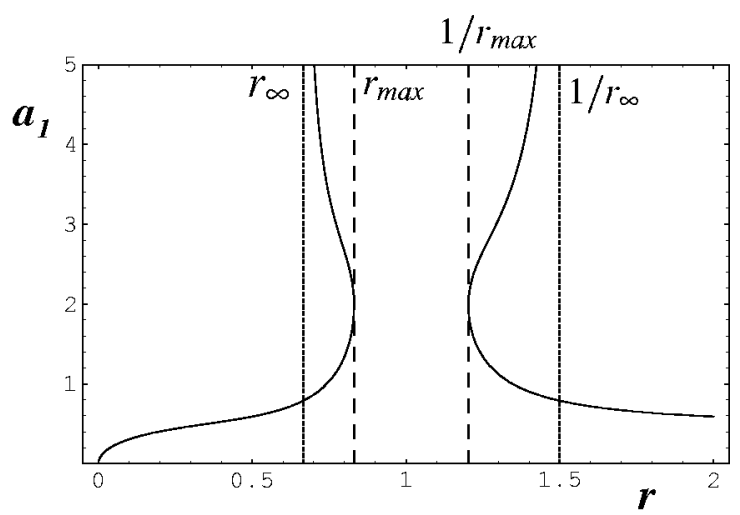

A)

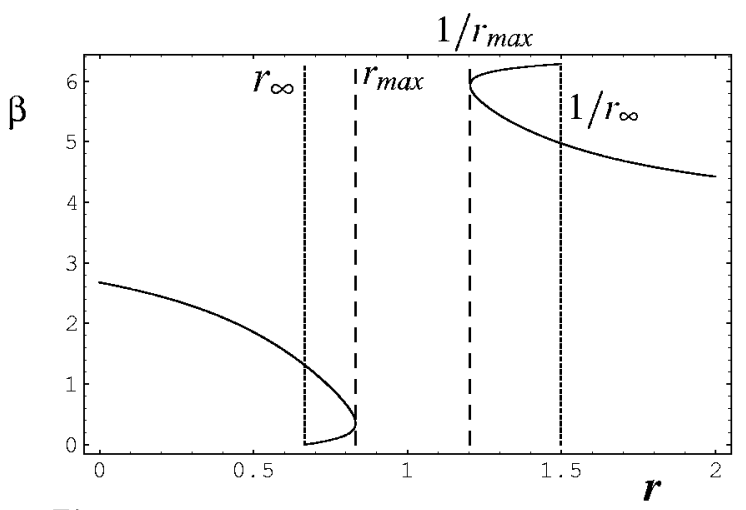

B)

FIG. 9. Projection on planes (A) $a_{1}-r$ and (B) $\beta-r$ of branches $\Lambda_{l}$ and $\Lambda_{h}$ of line of fixed points on invariant plane $a_{2}=0$, at $\Gamma=0$, given by Eqs. (24a) and $(24 b)$.

with the null value $\lambda_{3}$ corresponding to an eigenvector tangent to $\Lambda$. The eigenspace associated to $\lambda_{1}$ and $\lambda_{2}$ is tangent to the invariant plane $a_{1}=$ constant at the respective fixed point; as seen in (29), for flow on the space $a_{2}=0$, points on the branch $\Lambda_{l}$ are stable and points on $\Lambda_{h}$ are unstable. In each plane $a_{1}=a_{10}<a_{1}\left(r_{\max }\right)$ within the space $a_{2}=0$ the flow is determined by Eqs. (23c) and (23d), giving

$$
r \frac{d \beta}{d r}=\frac{a_{10}^{2}\left(1+r^{2}\right) \cos \beta-\left(2 \bar{V} a_{10}^{2}-\nu\right) r}{a_{10}^{2}\left(1-r^{2}\right) \sin \beta-\left(\gamma_{3}-\gamma_{2}\right) r} .
$$

One readily verifies that, except for particular separatrices, all trajectories start and end at the fixed points that are intersections of branches $\Lambda_{l}$ and $\Lambda_{h}$ with the $a_{10}$ plane (2D foci with the eigenvalues $\left.\lambda_{1,2}\right){ }^{17}$ Also, one can verify that once reached the line $r=1$ in that plane all trajectories keep $r$ less than unity. The entire flow in (30) tends to the stable focus at $r<1$. In the space $a_{2}=0$, the entire flow moves from the unstable branch $\Lambda_{h}$ to the stable branch $\Lambda_{l}$.

The eigenvalue for stability of $\Lambda$-points off the surface $a_{2}=0$, which is the factor multiplying $a_{2}$ in Eq. (23b), $\lambda_{4}$ $=-\gamma_{2}+r a_{1}^{2} \sin \beta$, can be rewritten using (24a) as

$$
\lambda_{4}=\frac{\gamma_{3} r^{2}-\gamma_{2}}{1-r^{2}}
$$

The associated eigenvector is transverse to the surface $a_{2}$ $=0$ (parallel to the $a_{2}$ axis). Equation (31) shows that, for motion off that surface, all points on the $\Lambda_{h}$ branch are stable, whereas only those points on the $\Lambda_{l}$ branch with

$$
r<\sqrt{\gamma_{2} / \gamma_{3}}
$$

if any, are stable. Hence, for the flow in the entire 4D space, the stable fixed points of $\Lambda$ are those on the $r<1$ branch satisfying condition (32).

Under condition $\sqrt{\gamma_{2} / \gamma_{3}}<r_{\max }$, reading as

$$
\bar{V}^{2}<\frac{\left(\gamma_{2}+\gamma_{3}\right)^{2}+\nu^{2}}{4 \gamma_{2} \gamma_{3}},
$$

there will be a point $P_{0}$ in the arc $a_{1}<a_{1}\left(r_{\max }\right)$ of $\Lambda_{l}$ having $\lambda_{4}=0$; points on that arc above $P_{0}$ [i.e., $\left.a_{1}>a_{1}\left(P_{0}\right)\right]$ will be unstable. Note that (33) recovers condition (16) for $\gamma_{3}$ $=\gamma_{2}$. Under the additional condition $r_{\infty}<\sqrt{\gamma_{2} / \gamma_{3}}$ there will also be a point $P_{0}^{*}$ in the arc $a_{1}>a_{1}\left(r_{\max }\right)$ of $\Lambda_{l}$ having $\lambda_{4}$ $=0$; points on that arc above $P_{0}^{*}$ will also be unstable. The full arc would be unstable in the opposite case, $\sqrt{\gamma_{2} / \gamma_{3}}$ $<r_{\infty}$, which reads as

$$
\bar{V}<\frac{1+\gamma_{2} / \gamma_{3}}{2 \sqrt{\gamma_{2} / \gamma_{3}}} .
$$

This condition cannot be satisfied with $\gamma_{3}=\gamma_{2}$, when it reads $\bar{V}<1$.

We may then conclude that, for $\Gamma=0$, and under conditions (33) and (34), the attractor of the flow is the $a_{1}$ $<a_{1}\left(r_{\max }\right) \Lambda_{l}$-arc below $P_{0}$ in the space $a_{2}=0$. Note that $\Lambda_{l}$ points above $P_{0}$ have an 1D unstable manifold transverse to $a_{2}=0$, corresponding to the positive sign of the eigenvalue $\lambda_{4}$. There are thus singular orbits that leave that surface at those points and end on the $\Lambda_{l}$-points below $P_{0}$, all of which have stable manifolds transverse to $a_{2}=0$ (and lie in the $r$ $<1$ domain). If the opposite of (34) holds, that is, if $P_{0}^{*}$ exists, the attractor includes the $a_{1}>a_{1}\left(r_{\max }\right), \Lambda_{l}$-arc above $P_{0}^{*}$, but singular orbits leaving the unstable arc still end at lower $a_{1}$ on the stable arc below $P_{0}$.

The full 3-wave model differs from the reduced 3-wave model, for $\Gamma=0$, in a number of significant phase-space features. There are sets of periodic orbits no longer; the line of fixed points $\Lambda$ covers the entire $0<a_{1}<\infty$ range; there may just exist one point on $\Lambda$ that is neutrally stable off the space $a_{3}=a_{2}=0$. Yet, the fundamental features leading to the hard transition in phase-space dynamics when $\Gamma$ is made positive, within some parametric subdomain, are common to both models. For $\Gamma=0$, the entire flow is asymptotic to the space $a_{3}=a_{2}=0$; there is a line of fixed points $\Lambda$ in that space with two branches having different stability character; one branch has an stable arc of fixed points at a low $a_{1}$ range and an arc unstable off $a_{3}=a_{2}=0$ at a higher $a_{1}$ range. These features are all that is needed for the hard transition to be present.

\section{DISCUSSION OF RESULTS}

We have truncated the derivative nonlinear Schrödinger (DNLS) equation describing the interaction of circularly polarized Alfvén waves of finite amplitude, to explore weakly nonlinear dynamics in the coherent cubic coupling of three 
waves near resonance (3WRI), wave 1 being linearly unstable and waves 2 and 3 damped. We have considered a broad scenario for chaos which several 3WRI systems, for both cubic and quadratic coupling, had exhibited: No matter how small the growth rate $\Gamma$ of the unstable wave there exists certain parametric domain with a fully developed attractor (chaotic in some subdomain) that is absent at $\Gamma \leqslant 0$. To explore the structural stability of this hard transition to complex phase-space dynamics we have considered both the reduced 3 -wave model (equal dampings of daughter waves, leading to a 3D flow for wave amplitudes $a_{1}, a_{2}$ and a relative phase) and the fully 3-wave model (different dampings, 4D flow). Structural stability (suggested by the appearance of that transition elsewhere as mentioned above) would be important because any 3 WRI model has limited validity.

Both reduced and full models showed the hard transition only occurring for left-hand circularly polarized waves, paralelling the known fact that LH time-harmonic solutions of the DNLS equation (for cold plasmas) are modulationally unstable, a case opposite $\mathrm{RH}$ polarized solutions. ${ }^{6}$ In the reduced model, transition occurs at damping less than about $0.25 \times(\text { wave- } 1 \text { frequency })^{2} /$ ion cyclotron frequency. A number of features determine the phase-space dynamics of the transition: For $\Gamma=0$, the entire flow is asymptotic to the space $a_{3}=a_{2}=0$, where a line of fixed points $\Lambda$ covers a limited $a_{1}$ range with periodic orbits below and above that range. A branch of $\Lambda$ has an arc of fixed points unstable off the space $a_{3}=a_{2}=0$, in between stable arcs; singular, heteroclinic orbits off the unstable arc return to that space at lower $a_{1}$. Chaotic attractors involve repeated slow rises on $\Lambda$, and possibly in the lower set of periodic orbits, followed by fast motion along the heteroclinic orbits. In the full 3 -wave model there are no sets of periodic orbits; the line of fixed points $\Lambda$ covers the entire $0<a_{1}<\infty$ range; there may exist just $1 \Lambda$-arc stable off the space $a_{3}=a_{2}=0$.

The easiest feature to detect in the transition would be the associated sudden break in $a_{2}$ behavior. The strict $\Gamma$ $\rightarrow 0^{+}$limit has theoretical interest but is impractical; during the slow rises on $\Lambda$, time would diverge and $a_{2}$ would drop below any noise level. ${ }^{15}$ The basic feature to consider in practical terms is that $a_{2}$ keeps null for negative $\Gamma$ whereas, at $\Gamma$ positive and finite, but small compared with dampings and with frequency mismatch, the ratio $a_{2} / a_{1}$ changes repeatedly from almost vanishing values during the long times on $\Lambda$ to values of order unity in the short times near heteroclinic orbits. Our scenario for chaos is a hard transition to relaxation oscillations that can be chaotic.

The DNLS equation has been used in relation to nonlinear MHD waves observed in the Earth's bow shock. ${ }^{28}$ In our model, the required growth rate $\Gamma$ could result, in general, from some plasma instability, due, say, to a beam-plasma interaction or to the plasma carrying a current that makes for negative Landau damping. The plasma does carry a current in the tether case. More to the point, however, tether signal detection would require modulating the current in the tether circuit, the Alfvén-radiation impedance being weak in the case of a steady tether current. ${ }^{1}$ The magnetic self-field of the tether ${ }^{3}$ would then result in a background magnetic field time modulated (at some frequency $\omega_{\text {mod }}$ ).
Such field may excite two growing Alfvén waves at half the modulation frequency and propagating along the magnetic field in opposite directions, ${ }^{29}$ each one representing the mother wave 1 of our problem. This would determine a definite wave number $k_{1} \approx \omega_{1} / V_{A}=\frac{1}{2} \omega_{\text {mod }} / V_{A}$. The ratio $k_{3} / k_{2}$, and thus both daughter wave numbers $k_{2}$ and $k_{3}=2 k_{1}$ $-k_{2}$, might then result from minimum-damping or maximum-amplitude considerations. A proper model may require, however, an analysis that includes more than a pair of daughter waves. It is here important that $d\left(a_{3} / a_{2}\right) / d t$ proved negative at $a_{3} / a_{2}=1$ in Sec. V; that made the surface $a_{2}$ $=0$ effectively invariant, which was essential for the transition. This suggests the transition could hold under multiplewave interaction, with the excited and less damped waves playing the fundamental role.

\section{ACKNOWLEDGMENTS}

This work was supported by Ministerio de Ciencia y Tecnologia of Spain, under Grant No. BFM01-3723. Work by S. Elaskar was supported by Ministerio de Ciencia y Tecnologia of Spain, under Grant No. SB01-0080.

${ }^{1}$ S. D. Drell, H. M. Foley, and M. A. Ruderman, J. Geophys. Res. 70, 3131 (1965); A. Barnett and S. Olbert, J. Geophys. Res., [Space Phys.] 91, 10117 (1986); J. R. Sanmartín and M. Martínez-Sanchez, ibid. 100, 1677 (1995).

${ }^{2}$ J. R. Sanmartin and R. D. Estes, J. Geophys. Res., [Space Phys.] 102, 14625 (1997).

${ }^{3}$ G. V. Khazanov, N. H. Stone, E. N. Krivorutsky, and E. W. Liemohn, J. Geophys. Res., [Space Phys.] 105, 15835 (2000); G. V. Khazanov, N. H. Stone, E. N. Krivorutsky, K. V. Gamayunov, and E. W. Liemohn, ibid. 106, 10565 (2001); J. R. Sanmartin and R. D. Estes, ibid. 107, SIA 2-1 (2002).

${ }^{4}$ J. R. Sanmartín and S. H. Lam, Phys. Fluids 14, 62 (1971).

${ }^{5}$ A. Rogister, Phys. Fluids 14, 2733 (1971); K. Mio, T. Ogino, K. Minami, and S. Takeda, J. Phys. Soc. Jpn. 41, 265 (1976).

${ }^{6}$ E. Mjølhus, J. Plasma Phys. 16, 321 (1976); 19, 437 (1978).

${ }^{7}$ D. J. Kaup and A. C. Newell, J. Math. Phys. 19, 798 (1978).

${ }^{8}$ S. R. Spangler and J. P. Sheerin, J. Plasma Phys. 27, 193 (1982); T. Hada, C. F. Kennel, and B. Buti, J. Geophys. Res., [Space Phys.] 94, 65 (1989); M. V. Medvedev and P. H. Diamond, Phys. Plasmas 3, 863 (1996); T. Passot and P. L. Sulem, ibid. 10, 3887 (2003).

${ }^{9}$ R. C. Davidson, Methods in Nonlinear Plasma Theory (Academic, New York, 1972), Chap. 6; A. Bers, in Plasma Physics-Les Houches 1972, edited by C. De Witt and J. Peyreaud (Gordon and Breach, New York, 1975), pp. 117-215; J. Weiland and H. Wilhelmsson, Coherent Nonlinear Interaction of Waves and Plasmas (Pergamon, New York, 1977).

${ }^{10}$ S. Ya. Vyshkind and M. I. Rabinovich, Sov. Phys. JETP 44, 292 (1976); A. S. Pikovsky and M. I. Rabinovich, Physica D 2, 8 (1981).

${ }^{11}$ J. M. Wersinger, J. M. Finn, and E. Ott, Phys. Rev. Lett. 44, 453 (1980); Phys. Fluids 23, 1142 (1980).

${ }^{12}$ M. N. Bussac, Phys. Rev. Lett. 49, 1939 (1982); C. Meunier, M. N. Bussac, and G. Laval, Physica D 4, 236 (1982).

${ }^{13}$ D. W. Hughes and M. R. E. Proctor, Physica D 46, 163 (1990).

${ }^{14}$ O. López-Rebollal and J. R. Sanmartín, Physica D 89, 204 (1995).

${ }^{15}$ E. del Río, J. R. Sanmartín, and O. López-Rebollal, Int. J. Bifurcation Chaos Appl. Sci. Eng. 8, 2255 (1998).

${ }^{16}$ D. W. Hughes and M. R. E. Proctor, Wave Motion 20, 201 (1994).

${ }^{17}$ O. López-Rebollal, J. R. Sanmartín, and E. del Río, Phys. Plasmas 5, 2861 (1998).

${ }^{18}$ M. I. Rabinovich and A. L. Fabrikant, Sov. Phys. JETP 50, 311 (1979); D. A. Russell and E. Ott, Phys. Fluids 24, 1976 (1981).

${ }^{19}$ J. R. Sanmartín, O. López-Rebollal, and N. de Paola, Physica D 69, 148 (1993).

${ }^{20}$ P. Terry and W. Horton, Phys. Fluids 25, 491 (1982); A. M. Martins and J. T. Mendonça, ibid. 31, 3286 (1988).

${ }^{21}$ P. A. Robinson and P. M. Drysdale, Phys. Rev. Lett. 77, 2698 (1996); P. M. Drysdale and P. A. Robinson, Phys. Plasmas 9, 4896 (2002).

${ }^{22}$ G. I. de Oliveira, L. P. L. de Oliveira, and F. B. Rizzato, Physica D 104, 119 (1997). 
${ }^{23}$ C. C. Chow, Physica D 81, 237 (1995).

${ }^{24}$ C. S. Kueny and P. J. Morrison, Phys. Plasmas 2, 1926 (1995); 2, 4149 (1995).

${ }^{25}$ A. C. L. Chian, S. R. Lopes, and J. R. Abaldo, Physica D 99, 269 (1996); B. Lefebvre and V. Krasnoselskikh, ibid. 152-153, 742 (2001).

${ }^{26}$ S. Rauf and J. A. Tataronis, Phys. Plasmas 2, 1453 (1995); J. Plasma Phys. 55, 173 (1996).

${ }^{27}$ A. I. Akhiezer, I. A. Akhiezer, R. V. Polovin, A. G. Sitenko, and K. N.
Stepanov, Plasma Electrodynamics (Pergamon, Oxford, 1975), Vol. 1, p. 250.

${ }^{28}$ S. R. Spangler, in Nonlinear Waves and Chaos in Space Plasmas, edited by T. Hada and H. Matsumoto (Terrapub, Tokyo, 1997), p. 171; E. Mjølhus and T. Hada, ibid. p. 121.

${ }^{29}$ G. Vahala and D. Montgomery, Phys. Fluids 14, 1137 (1971); N. F. Cramer, Plasma Phys. 17, 967 (1975); M. P. Hertzberg, N. F. Cramer, and S. V. Vladimirov, Phys. Plasmas 10, 3160 (2003). 
Physics of Plasmas is copyrighted by the American Institute of Physics (AIP). Redistribution of journal material is subject to the AIP online journal license and/or AIP copyright. For more information, see http:/ojps.aip.org/pop/popcr.jsp

Copyright of Physics of Plasmas is the property of American Institute of Physics and its content may not be copied or emailed to multiple sites or posted to a listserv without the copyright holder's express written permission. However, users may print, download, or email articles for individual use. 